\title{
Bounded Toeplitz and Hankel products on weighted Bergman spaces of the unit ball
}

\author{
by Matgorzata Michalska, Maria Nowak \\ and PaWee SobOleWski (Lublin)
}

\begin{abstract}
We prove a sufficient condition for products of Toeplitz operators $T_{f} T_{\bar{g}}$, where $f, g$ are square integrable holomorphic functions in the unit ball in $\mathbb{C}^{n}$, to be bounded on the weighted Bergman space. This condition slightly improves the result obtained by K. Stroethoff and D. Zheng. The analogous condition for boundedness of products of Hankel operators $H_{f} H_{g}^{*}$ is also given.
\end{abstract}

1. Introduction. Let $d v(z)$ denote the Lebesgue measure on the unit ball $\mathbb{B}$ in $\mathbb{C}^{n}$ normalized so that $\int_{\mathbb{B}} d v=1$. For $\alpha>-1$ let

$$
d v_{\alpha}(z)=c_{\alpha}\left(1-|z|^{2}\right)^{\alpha} d v(z)
$$

where $c_{\alpha}=\Gamma(n+1+\alpha) /(n ! \Gamma(\alpha+1))$, denote the weighted Lebesgue measure on the unit ball. The Bergman space $A_{\alpha}^{2}$ is the Hilbert space consisting of holomorphic functions on $\mathbb{B}$ for which

$$
\|f\|=\|f\|_{\alpha}=\left(\int_{\mathbb{B}}|f(z)|^{2} d v_{\alpha}(z)\right)^{1 / 2}<\infty .
$$

Let $P_{\alpha}$ denote the orthogonal projection from $L^{2}\left(\mathbb{B}, d v_{\alpha}\right)$ onto $A_{\alpha}^{2}$. For $f \in$ $L^{2}\left(\mathbb{B}, d v_{\alpha}\right)$, the Toeplitz operator $T_{f}$ and the Hankel operator $H_{f}$ with symbol $f$ are defined densely on the space $A_{\alpha}^{2}$ by $T_{f}(h)=P_{\alpha}(f h)$ and $H_{f}(h)=$ $f h-P_{\alpha}(f h)$, respectively. The Bergman space $A_{\alpha}^{2}$ has the reproducing kernel $K_{w}^{(\alpha)}$ given by

$$
K_{w}^{(\alpha)}(z)=\frac{1}{(1-\langle z, w\rangle)^{n+\alpha+1}}, \quad z, w \in \mathbb{B}
$$

so for $h \in A_{\alpha}^{2}$ we have

$$
h(w)=\left\langle h, K_{w}^{(\alpha)}\right\rangle_{\alpha}=\int_{\mathbb{B}} \frac{h(z)}{(1-\langle w, z\rangle)^{n+\alpha+1}} d v_{\alpha}(z),
$$

2010 Mathematics Subject Classification: Primary 47B35; Secondary 32A36.

Key words and phrases: Toeplitz operator, Hankel operator, weighted Bergman spaces. 
and for $f \in L^{2}\left(\mathbb{B}, d v_{\alpha}\right)$,

$$
P_{\alpha} f(w)=\left\langle f, K_{w}^{(\alpha)}\right\rangle_{\alpha}=\int_{\mathbb{B}} \frac{f(z)}{(1-\langle w, z\rangle)^{n+\alpha+1}} d v_{\alpha}(z) .
$$

We will denote the normalized reproducing kernel for $A_{\alpha}^{2}$ by

$$
k_{w}^{(\alpha)}(z)=\frac{\left(1-|w|^{2}\right)^{(n+\alpha+1) / 2}}{(1-\langle z, w\rangle)^{n+\alpha+1}}, \quad z, w \in \mathbb{B} .
$$

In their recent papers [7] and [8] K. Stroethoff and D. Zheng studied the products of Toeplitz operators $T_{f} T_{\bar{g}}$, where $f, g \in A_{\alpha}^{2}$, densely defined on $A_{\alpha}^{2}$. To state their results we need the following notation. For $w \in \mathbb{B}$ let $\varphi_{w}$ be the automorphism of $\mathbb{B}$ of the form

$$
\varphi_{w}(z)=\frac{w-P_{w}(z)-s_{w} Q_{w}(z)}{1-\langle z, w\rangle}
$$

where $s_{w}=(1-|w|)^{1 / 2}, P_{w}(z)=\frac{\langle z, w\rangle}{|w|^{2}} w$ if $w \neq 0, P_{0}(z)=0$ and $Q_{w}=I-P_{w}$ (see, e.g., [4], 10] for definition and properties of the automorphism group of $\mathbb{B})$.

For $u \in L^{1}\left(\mathbb{B}, d v_{\alpha}\right)$ and $w \in \mathbb{B}$ define

$$
B[u](w)=B^{(\alpha)}[u](w)=\int_{\mathbb{B}} u \circ \varphi_{w}(z) d v_{\alpha}(z)=\int_{\mathbb{B}} u(z)\left|k_{w}^{(\alpha)}(z)\right|^{2} d v_{\alpha}(z) .
$$

Stroethoff and Zheng obtained the following results for the Toeplitz products.

Theorem 1.1 ([7], [8]). Let $-1<\alpha<\infty$, and let $f$ and $g$ be in $A_{\alpha}^{2}$. If $T_{f} T_{\bar{g}}$ is bounded on $A_{\alpha}^{2}$, then

$$
\sup _{w \in \mathbb{B}} B\left[|f|^{2}\right](w) B\left[|g|^{2}\right](w)<\infty .
$$

Theorem $1.2([7],[8])$. Let $-1<\alpha<\infty$, and let $f$ and $g$ be in $A_{\alpha}^{2}$. If for $\varepsilon>0$,

$$
\sup _{w \in \mathbb{B}} B\left[|f|^{2+\varepsilon}\right](w) B\left[|g|^{2+\varepsilon}\right](w)<\infty,
$$

then $T_{f} T_{\bar{g}}$ is bounded on $A_{\alpha}^{2}$.

In their earlier paper Stroethoff and Zheng [6] also studied the product of Hankel operators $H_{f} H_{g}^{*}, f, g \in L^{2}(\mathbb{D}, d A)$, densely defined on $\left(A^{2}\right)^{\perp}$ in the setting of the unit disk. Recently, the analogous result for the unit ball has been obtained by Lu and Liu in [2]. More exactly they proved the following.

Theorem 1.3 ([2], [6]). Let $-1<\alpha<\infty$, and let $f$ and $g$ be in $L^{2}\left(\mathbb{B}, d v_{\alpha}\right)$. If $H_{f} H_{g}^{*}$ is bounded, then

$$
\sup _{w \in \mathbb{B}}\left\|f \circ \varphi_{w}-P_{\alpha}\left(f \circ \varphi_{w}\right)\right\|\left\|g \circ \varphi_{w}-P_{\alpha}\left(g \circ \varphi_{w}\right)\right\|<\infty .
$$


Theorem 1.4 ([2], [6]). Let $-1<\alpha<\infty$, and let $f$ and $g$ be in $L^{2}\left(\mathbb{B}, d v_{\alpha}\right)$. If there is $\varepsilon>0$ such that

$$
\sup _{w \in \mathbb{B}}\left\|f \circ \varphi_{w}-P_{\alpha}\left(f \circ \varphi_{w}\right)\right\|_{2+\varepsilon}\left\|g \circ \varphi_{w}-P_{\alpha}\left(g \circ \varphi_{w}\right)\right\|_{2+\varepsilon}<\infty,
$$

then $H_{f} H_{g}^{*}$ is bounded.

The above-cited results are analogous to those obtained earlier for the Hardy space $H^{2}$ (e.g., [1], [5], 9]).

Also we mention that generalizations of Theorems 1.1 and 1.2 have been obtained by J. Miao in [3].

In this paper we give a sufficient condition for boundedness of Toeplitz products which is slightly weaker than that given in Theorem 1.2. We also obtain a similar condition for Hankel products in the setting of the unit ball that is slightly weaker than Theorem 1.4 .

2. Results. For $\varepsilon>0, w \in \mathbb{B}$ and $u \in L^{1}\left(\mathbb{B}, d v_{\alpha}\right)$, set

$$
B_{\varepsilon}[u](w)=B_{\varepsilon}^{(\alpha)}[u](w)=\int_{\mathbb{B}} u \circ \varphi_{w}(z) \log ^{1+\varepsilon}\left(\frac{1}{1-|z|}\right) d v_{\alpha}(z) .
$$

We will prove the following.

Theorem 2.1. Let $-1<\alpha<\infty$, and let $f, g \in A_{\alpha}^{2}$. If there is an $\varepsilon>0$ such that

$$
\sup _{w \in \mathbb{B}} B_{\varepsilon}\left[|f|^{2}\right](w) B_{\varepsilon}\left[|g|^{2}\right](w)<\infty,
$$

then the Toeplitz product $T_{f} T_{\bar{g}}$ is bounded on $A_{\alpha}^{2}$.

Theorem 2.2. Let $-1<\alpha<\infty$, and let $f, g \in L^{2}\left(\mathbb{B}, d v_{\alpha}\right)$. If there is an $\varepsilon>0$ such that

$$
\begin{aligned}
\sup _{w \in \mathbb{B}} \|\left[f \circ \varphi_{w}-\right. & \left.P_{\alpha}\left(f \circ \varphi_{w}\right)\right] \log (1+\varepsilon) / 2\left(\frac{1}{1-|z|}\right) \| \\
\times & \left\|\left[g \circ \varphi_{w}-P_{\alpha}\left(g \circ \varphi_{w}\right)\right] \log ^{(1+\varepsilon) / 2}\left(\frac{1}{1-|z|}\right)\right\|<\infty,
\end{aligned}
$$

then the operator $H_{f} H_{g}^{*}$ is bounded on $\left(A_{\alpha}^{2}\right)^{\perp}$.

For a multi-index $\nu=\left(\nu_{1}, \ldots, \nu_{n}\right)$ such that $|\nu|=\nu_{1}+\cdots+\nu_{n}=m$ and $f$ holomorphic in $\mathbb{B}$ define

$$
D^{\nu} f=\frac{\partial f^{m}}{\partial z_{1}^{\nu_{1}} \ldots \partial z_{n}^{\nu_{n}}} .
$$

In the proofs of the above stated theorems we will use the following lemma.

Lemma 2.3. Assume that $-1<\alpha<\infty, n \geq 2, \varepsilon>0$ and $\nu$ is a multi-index such that $|\nu|=m$. Then 
(a) for every $f, h \in A_{\alpha}^{2}$ and $w \in \mathbb{B}$,

$$
\begin{aligned}
\left|\left(D^{\nu} T_{\bar{f}}\right) h(w)\right| \leq & \frac{C\left\{B_{\varepsilon}\left[|f|^{2}\right](w)\right\}^{1 / 2}}{\left(1-|w|^{2}\right)^{(n+m+\alpha+1) / 2}} \\
& \times\left\{\int_{\mathbb{B}} \frac{|h(z)|^{2}}{|1-\langle w, z\rangle|^{m}} \log ^{-(1+\varepsilon)}\left(\frac{1}{1-\left|\varphi_{w}(z)\right|}\right) d v_{\alpha}(z)\right\}^{1 / 2},
\end{aligned}
$$

(b) for $g \in L^{2}\left(\mathbb{B}, d v_{\alpha}\right), u \in\left(A_{\alpha}^{2}\right)^{\perp}$ and $w \in \mathbb{B}$,

$$
\begin{aligned}
\left|\left(D^{\nu} H_{g}^{*} u\right)(w)\right| \leq & \frac{C}{\left(1-|w|^{2}\right)^{(n+m+\alpha+1) / 2}} \\
& \times\left\|\left(g \circ \varphi_{w}-P_{\alpha}\left(g \circ \varphi_{w}\right)\right) \log ^{(1+\varepsilon) / 2}\left(\frac{1}{1-|z|}\right)\right\| \\
& \times\left\{\int_{\mathbb{B}} \frac{|u(z)|^{2}}{|1-\langle w, z\rangle|^{m}} \log ^{-(1+\varepsilon)}\left(\frac{1}{1-\left|\varphi_{w}(z)\right|}\right) d v_{\alpha}(z)\right\}^{1 / 2} .
\end{aligned}
$$

Proof. (a) Since for any multi-index $\nu$ with $|\nu|=m$ we have

$$
\left(D^{\nu} T_{\bar{f}} h\right)(w)=\frac{\Gamma(n+m+\alpha+1)}{\Gamma(n+\alpha+1)} \int_{\mathbb{B}} \frac{\overline{z^{\nu} f(z)} h(z)}{(1-\langle w, z\rangle)^{n+m+\alpha+1}} d v_{\alpha}(z),
$$

by the Cauchy-Schwarz inequality we get

$$
\begin{aligned}
& \left|\left(D^{\nu} T_{\bar{f}} h\right)(w)\right| \leq \frac{C}{\left(1-|w|^{2}\right)^{(n+\alpha+1) / 2}} \\
& \quad \times\left\{\int_{\mathbb{B}} \frac{|f(z)|^{2}\left(1-|w|^{2}\right)^{n+\alpha+1}}{|1-\langle w, z\rangle|^{2 n+2 \alpha+2+m}} \log ^{1+\varepsilon}\left(\frac{1}{1-\left|\varphi_{w}(z)\right|}\right) d v_{\alpha}(z)\right\}^{1 / 2} \\
& \quad \times\left\{\int_{\mathbb{B}} \frac{|h(z)|^{2}}{|1-\langle w, z\rangle|^{m}} \log ^{-(1+\varepsilon)}\left(\frac{1}{1-\left|\varphi_{w}(z)\right|}\right) d v_{\alpha}(z)\right\}^{1 / 2} \\
& =\frac{C\left\{B_{\varepsilon}\left[|f|^{2}\right](w)\right\}^{1 / 2}}{\left(1-|w|^{2}\right)^{(n+m+\alpha+1) / 2}}\left\{\int_{\mathbb{B}} \frac{|h(z)|^{2}}{|1-\langle w, z\rangle|^{m}} \log ^{-(1+\varepsilon)}\left(\frac{1}{1-\left|\varphi_{w}(z)\right|}\right) d v_{\alpha}(z)\right\}^{1 / 2},
\end{aligned}
$$

where the last equality follows from the change-of-variable formula.

(b) Since

$$
h(z)=\frac{z^{\nu} P_{\alpha}\left(g \circ \varphi_{w}\right) \circ \varphi_{w}(z)}{(1-\langle z, w\rangle)^{n+m+\alpha+1}} \in A_{\alpha}^{2},
$$

for $u \in\left(A_{\alpha}^{2}\right)^{\perp}$ we have

$$
\langle u, h\rangle_{\alpha}(w)=\int_{\mathbb{B}} \frac{u(z) \overline{z^{\nu} P_{\alpha}\left(g \circ \varphi_{w}\right) \circ \varphi_{w}(z)}}{(1-\langle w, z\rangle)^{n+m+\alpha+1}} d v_{\alpha}(z) \equiv 0 .
$$


Consequently,

$$
\begin{aligned}
& \left|\left(D^{\nu} H_{g}^{*} u\right)(w)\right|=\mid\left(D^{\nu} P_{\alpha}(\bar{g} u)(w)-\langle u, h\rangle_{\alpha}(w) \mid\right. \\
& =\left|\frac{\Gamma(n+m+\alpha+1)}{\Gamma(n+\alpha+1)} \int_{\mathbb{B}} \frac{\overline{z^{\nu} g(z)} u(z)}{(1-\langle w, z\rangle)^{n+m+\alpha+1}} d v_{\alpha}(z)-\langle u, h\rangle_{\alpha}(w)\right| \\
& \leq \frac{C}{\left(1-|w|^{2}\right)^{(n+\alpha+1) / 2}}\left\{\int_{\mathbb{B}} \frac{|u(z)|^{2}}{|1-\langle w, z\rangle|^{m}} \log ^{-(1+\varepsilon)}\left(\frac{1}{1-\left|\varphi_{w}(z)\right|}\right) d v_{\alpha}(z)\right\}^{1 / 2} \\
& \times\left\{\int_{\mathbb{B}} \frac{\left|g(z)-\left(P_{\alpha}\left(g \circ \varphi_{w}\right) \circ \varphi_{w}\right)(z)\right|^{2}\left(1-|w|^{2}\right)^{n+\alpha+1}}{|1-\langle w, z\rangle|^{2 n+2 \alpha+2+m}}\right. \\
& \left.\times \log ^{1+\varepsilon}\left(\frac{1}{1-\left|\varphi_{w}(z)\right|}\right) d v_{\alpha}(z)\right\}^{1 / 2} \\
& =\frac{C}{\left(1-|w|^{2}\right)^{(n+m+\alpha+1) / 2}}\left\|\left(g \circ \varphi_{w}-P_{\alpha}\left(g \circ \varphi_{w}\right)\right) \log (1+\varepsilon) / 2\left(\frac{1}{1-|z|}\right)\right\| \\
& \times\left\{\int_{\mathbb{B}} \frac{|u(z)|^{2}}{|1-\langle w, z\rangle|^{m}} \log ^{-(1+\varepsilon)}\left(\frac{1}{1-\left|\varphi_{w}(z)\right|}\right) d v_{\alpha}(z)\right\}^{1 / 2} .
\end{aligned}
$$

In the case when $n=1$ the unit ball is the unit disk $\mathbb{D}$ of the complex plane. In this setting one can prove the following analogous result.

Lemma 2.4. Let $-1<\alpha<\infty$ and $\varepsilon>0$. Then

(a) for every $f, h \in A_{\alpha}^{2}$ and $w \in \mathbb{D}$,

$$
\begin{aligned}
\left|\left(T_{\bar{f}} h\right)^{\prime}(w)\right| \leq & \frac{C\left\{B_{\varepsilon}\left[|f|^{2}\right](w)\right\}^{1 / 2}}{\left(1-|w|^{2}\right)^{(\alpha+2) / 2}} \\
& \times\left\{\int_{\mathbb{D}} \frac{|h(z)|^{2}}{|1-\bar{z} w|^{2}} \log ^{-(1+\varepsilon)}\left(\frac{1}{1-\left|\varphi_{w}(z)\right|}\right) d v_{\alpha}(z)\right\}^{1 / 2},
\end{aligned}
$$

(b) for $g \in L^{2}\left(\mathbb{D}, d v_{\alpha}\right), u \in\left(A_{\alpha}^{2}\right)^{\perp}$ and $w \in \mathbb{D}$,

$$
\begin{aligned}
\left|\left(H_{g}^{*} u\right)^{\prime}(w)\right| \leq & \frac{C}{\left(1-|w|^{2}\right)^{(\alpha+2) / 2}}\left\|\left(g \circ \varphi_{w}-P_{\alpha}\left(g \circ \varphi_{w}\right)\right) \log ^{(1+\varepsilon) / 2}\left(\frac{1}{1-|z|}\right)\right\| \\
& \times\left\{\int_{\mathbb{D}} \frac{|u(z)|^{2}}{|1-\bar{z} w|^{2}} \log ^{-(1+\varepsilon)}\left(\frac{1}{1-\left|\varphi_{w}(z)\right|}\right) d v_{\alpha}(z)\right\}^{1 / 2} .
\end{aligned}
$$

Proof of Theorem 2.1. Clearly, we can assume that $0<\varepsilon<1$. We will show that for $u, v \in A_{\alpha}^{2}$,

$$
\left|\left\langle T_{f} T_{\bar{g}} u, v\right\rangle_{\alpha}\right| \leq C\|u\|\|v\| \text {. }
$$

We assume first that $n \geq 2$. By formula (4.11) in [8] for any positive integer $m$ there exist complex numbers $a_{j}, j=1, \ldots, 2 m-1$, and $b_{j}, j=1, \ldots, m$, 
such that the above inner product can be written as $I+I I+I I I$, where

$$
\begin{aligned}
I & =\frac{\Gamma(\alpha+1)}{\Gamma(\alpha+2 m+1)} \sum_{|\nu|=m \mathbb{B}} \int_{\mathbb{B}}\left(1-|w|^{2}\right)^{2 m}\left(D^{\nu} T_{\bar{g}} u\right)(w) \overline{\left(D^{\nu} T_{\bar{f}} v\right)(w)} d v_{\alpha}(w), \\
I I & =\sum_{j=1}^{2 m-1} a_{j} \sum_{|\nu|=m \mathbb{B}} \int_{\mathbb{B}}\left(1-|w|^{2}\right)^{2 m+j}\left(D^{\nu} T_{\bar{g}} u\right)(w) \overline{\left(D^{\nu} T_{\bar{f}} v\right)(w)} d v_{\alpha}(w), \\
I I I & =\sum_{j=1}^{m} b_{j} \int_{\mathbb{B}}\left(1-|w|^{2}\right)^{2 m+j-1}\left(T_{\bar{g}} u\right)(w) \overline{\left(T_{\bar{f}} v\right)(w)} d v_{\alpha}(w) .
\end{aligned}
$$

If we assume that $m \geq n+1$, then by (a) in Lemma 2.3 and the CauchySchwarz inequality, we obtain

$$
\begin{aligned}
|I I| \leq & C|I| \leq C \int_{\mathbb{B}} \frac{\left\{B_{\varepsilon}\left[|f|^{2}\right](w)\right\}^{1 / 2}}{\left(1-|w|^{2}\right)^{n+1-m+\alpha}} \\
& \times\left\{\int_{\mathbb{B}} \frac{|u(z)|^{2}}{|1-\langle z, w\rangle|^{m}} \log ^{-(1+\varepsilon)}\left(\frac{1}{1-\left|\varphi_{w}(z)\right|}\right) d v_{\alpha}(z)\right\}^{1 / 2} \\
& \times\left\{B_{\varepsilon}\left[|g|^{2}\right](w)\right\}^{1 / 2} \\
& \times\left\{\int_{\mathbb{B}} \frac{|v(z)|^{2}}{|1-\langle z, w\rangle|^{m}} \log ^{-(1+\varepsilon)}\left(\frac{1}{1-\left|\varphi_{w}(z)\right|}\right) d v_{\alpha}(z)\right\}^{1 / 2} d v_{\alpha}(w) \\
\leq & C \sup _{w \in \mathbb{B}}\left\{B_{\varepsilon}\left[|f|^{2}\right](w) B_{\varepsilon}\left[|g|^{2}\right](w)\right\}^{1 / 2} \\
& \times\left\{\int_{\mathbb{B} \mathbb{B}} \frac{|u(z)|^{2}}{|1-\langle z, w\rangle|^{m}} \log ^{-(1+\varepsilon)}\left(\frac{1}{1-\left|\varphi_{w}(z)\right|}\right) d v_{\alpha}(z) d v(w)\right\}^{1 / 2} \\
& \times\left\{\int_{\mathbb{B} \mathbb{B}} \frac{|v(z)|^{2}}{|1-\langle z, w\rangle|^{m}} \log ^{-(1+\varepsilon)}\left(\frac{1}{1-\left|\varphi_{w}(z)\right|}\right) d v_{\alpha}(z) d v(w)\right\}^{1 / 2} .
\end{aligned}
$$

Now we will show that if $m<n+2$, then

$$
I_{1}=\int_{\mathbb{B} \mathbb{B}} \frac{|u(z)|^{2}}{|1-\langle z, w\rangle|^{m}} \log ^{-(1+\varepsilon)}\left(\frac{1}{1-\left|\varphi_{w}(z)\right|}\right) d v_{\alpha}(z) d v(w) \leq C\|u\|_{2}^{2} .
$$

Fubini's theorem and the change of variable $w^{\prime}=\varphi_{z}(w)$ give

$$
\begin{aligned}
I_{1}= & \int_{\mathbb{B}} \int_{\mathbb{B}} \frac{|u(z)|^{2}}{|1-\langle z, w\rangle|^{m}} \log ^{-(1+\varepsilon)}\left(\frac{1}{1-\left|\varphi_{w}(z)\right|}\right) d v_{\alpha}(z) d v(w) \\
= & \int_{\mathbb{B}}|u(z)|^{2}\left(1-|z|^{2}\right)^{n+1-m} \\
& \times \int_{\mathbb{B}} \frac{1}{\left|1-\left\langle z, w^{\prime}\right\rangle\right|^{2 n+2-m}} \log ^{-(1+\varepsilon)}\left(\frac{1}{1-\left|w^{\prime}\right|}\right) d v\left(w^{\prime}\right) d v_{\alpha}(z)
\end{aligned}
$$




$$
\begin{aligned}
= & \int_{\mathbb{B}}|u(z)|^{2}\left(1-|z|^{2}\right)^{n+1-m}\left(2 n \int_{0}^{1} r^{2 n-1} \log ^{-(1+\varepsilon)}\left(\frac{1}{1-r}\right)\right. \\
& \left.\times \int_{S} \frac{1}{|1-\langle r \zeta, z\rangle|^{2 n+2-m}} d \sigma(\zeta) d r\right) d v_{\alpha}(z) .
\end{aligned}
$$

By Theorem 1.12 in [10],

$$
\int_{S} \frac{1}{|1-\langle r \zeta, z\rangle|^{2 n+2-m}} d \sigma(\zeta) \leq \frac{C}{(1-r|z|)^{n+2-m}} \leq \frac{C}{(1-|z|)^{n+1-m}} \frac{1}{1-r} .
$$

Thus

$$
I_{1} \leq C \int_{\mathbb{B}}|u(z)|^{2} d v_{\alpha}(z) \int_{0}^{1} \log ^{-(1+\varepsilon)}\left(\frac{1}{1-r}\right) \frac{r}{1-r} d r .
$$

To see that the last integral converges for $0<\varepsilon<1$, one can write

$$
\int_{0}^{1} \log ^{-(1+\varepsilon)}\left(\frac{1}{1-r}\right) \frac{r}{1-r} d r=\int_{0}^{1} t^{-1-\varepsilon}\left(1-e^{-t}\right) d t+\int_{1}^{+\infty} t^{-1-\varepsilon}\left(1-e^{-t}\right) d t .
$$

To obtain the same estimate for $|I I I|$ it is enough to observe that

$$
\begin{aligned}
&\left|\left(T_{\bar{f}} u\right)(w)\right|=\left|\int_{\mathbb{B}} \frac{\overline{f(z)} u(z)}{(1-\langle z, w\rangle)^{n+\alpha+1}} d v_{\alpha}(z)\right| \leq \int_{\mathbb{B}} \frac{|f(z)||u(z)|}{|1-\langle z, w\rangle|^{n+\alpha+1}} d v_{\alpha}(z) \\
&=\int_{\mathbb{B}} \frac{|f(z)||u(z)|}{|1-\langle z, w\rangle|^{(n+2 \alpha+1) / 2}|1-\langle z, w\rangle|^{(n+1) / 2}} \\
& \quad \times \log ^{(1+\varepsilon) / 2}\left(\frac{1}{1-\left|\varphi_{w}(z)\right|}\right) \log ^{-(1+\varepsilon) / 2}\left(\frac{1}{1-\left|\varphi_{w}(z)\right|}\right) d v_{\alpha}(z) \\
& \leq \frac{1}{\left(1-|w|^{2}\right)^{(n+\alpha+1) / 2}}\left\{\int_{\mathbb{B}} \frac{|f(z)|^{2}\left(1-|w|^{2}\right)^{n+\alpha+1}|1-\langle z, w\rangle|^{n+1}}{|1-\langle z, w\rangle|^{2 n+2 \alpha+2}}\right. \\
&\left.\quad \times \log ^{(1+\varepsilon)}\left(\frac{1}{1-\left|\varphi_{w}(z)\right|}\right) d v_{\alpha}(z)\right\}^{1 / 2} \\
& \quad \times\left\{\int_{\mathbb{B}} \frac{|u(z)|^{2}}{|1-\langle z, w\rangle|^{n+1}} \log ^{-(1+\varepsilon)}\left(\frac{1}{1-\left|\varphi_{w}(z)\right|}\right) d v_{\alpha}(z)\right\}^{1 / 2} \\
& \leq \frac{C\left\{B_{\varepsilon}|f|^{2}(w)\right\}^{1 / 2}}{\left(1-|w|^{2}\right)^{(n+\alpha+1) / 2}}\left\{\int_{\mathbb{B}} \frac{|u(z)|^{2}}{|1-\langle z, w\rangle|^{n+1}} \log ^{-(1+\varepsilon)}\left(\frac{1}{1-\left|\varphi_{w}(z)\right|}\right) d v_{\alpha}(z)\right\}^{1 / 2} .
\end{aligned}
$$

In the case $n=1$, by formula (3.5) in [7] the inner product $\left\langle T_{f} T_{\bar{g}} u, v\right\rangle_{\alpha}$ is equal to $I+I I+I I I$, where

$$
I=\frac{\alpha+3}{\alpha+1} \int_{\mathbb{D}}\left(1-|w|^{2}\right)^{2}\left(T_{\bar{g}} u\right)(w) \overline{\left(T_{\bar{f}} v\right)(w)} d v_{\alpha}(w),
$$




$$
\begin{gathered}
I I=\frac{1}{(\alpha+1)(\alpha+2)} \int_{\mathbb{D}}\left(1-|w|^{2}\right)^{2}\left(T_{\bar{g}}^{\prime} u\right)(w) \overline{\left(T_{\bar{f}}^{\prime} v\right)(w)} d v_{\alpha}(w), \\
I I I=\frac{1}{(\alpha+1)(\alpha+3)} \int_{\mathbb{D}}\left(1-|w|^{2}\right)^{3}\left(T_{\bar{g}}^{\prime} u\right)(w) \overline{\left(T_{\bar{f}}^{\prime} v\right)(w)} d v_{\alpha}(w) .
\end{gathered}
$$

In view of Lemma 2.4 one can proceed analogously.

In view of part (b) in Lemmas 2.3 and 2.4. Theorem 2.2 can be proved in much the same way.

It follows from the next lemma that Theorem 2.1 contains Theorem 1.1.

Lemma 2.5. Let $-1<\alpha<\infty$ and let $f, g \in A_{\alpha}^{2}$. Then for $\varepsilon>0$ and for $w \in \mathbb{B}$,

$$
B_{\varepsilon}\left[|f|^{2}\right](w) B_{\varepsilon}\left[|g|^{2}\right](w) \leq C \cdot B\left[|f|^{2+\varepsilon}\right](w) B\left[|g|^{2+\varepsilon}\right](w) .
$$

Proof. Let $-1<\alpha<\infty, \varepsilon>0$ and $w \in \mathbb{B}$. By Hölder's inequality we get

$$
\begin{aligned}
B_{\varepsilon}\left[|f|^{2}\right] & (w)=\int_{\mathbb{B}}|f(z)|^{2} \log ^{1+\varepsilon}\left(\frac{1}{1-\left|\varphi_{w}(z)\right|}\right) \frac{\left(1-|w|^{2}\right)^{n+\alpha+1}}{|1-\langle w, z\rangle|^{2 n+2 \alpha+2}} d v_{\alpha}(z) \\
\leq & \left\{\int_{\mathbb{B}}|f(z)|^{2+\varepsilon} \frac{\left(1-|w|^{2}\right)^{n+\alpha+1}}{|1-\langle w, z\rangle|^{2 n+2 \alpha+2}} d v_{\alpha}(z)\right\}^{2 /(2+\varepsilon)} \\
& \times\left\{\int_{\mathbb{B}} \log ^{(1+\varepsilon)(2+\varepsilon) / \varepsilon}\left(\frac{1}{1-\left|\varphi_{w}(z)\right|}\right) \frac{\left(1-|w|^{2}\right)^{n+\alpha+1}}{|1-\langle w, z\rangle|^{2 n+2 \alpha+2}} d v_{\alpha}(z)\right\}^{\varepsilon /(2+\varepsilon)} \\
= & \left\{B\left[|f|^{2+\varepsilon}\right](w)\right\}^{2 /(2+\varepsilon)}\left\{\int_{\mathbb{B}} \log ^{(1+\varepsilon)(2+\varepsilon) / \varepsilon}\left(\frac{1}{1-|z|}\right) d v_{\alpha}(z)\right\}^{\varepsilon /(2+\varepsilon)} .
\end{aligned}
$$

Since the last integral is convergent, our claim follows.

Similarly one can prove that Theorem 2.2 contains Theorem 1.4

\section{References}

[1] D. Cruz-Uribe, The invertibility of the product of unbounded Toeplitz operators, Integral Equations Operator Theory 20 (1994), 231-237.

[2] Y. F. Lu and C. M. Liu, Toeplitz and Hankel products on Bergman spaces of the unit ball, Chin. Ann. Math. Ser. B 30 (2009), 293-310.

[3] J. Miao, Bounded Toeplitz products on the weighted Bergman spaces of the unit ball, J. Math. Anal. Appl. 346 (2008), 305-313.

[4] W. Rudin, Function Theory in the Unit Ball of $\mathbb{C}^{n}$, Grundlehren Math. Wiss. 241, Springer, Berlin, 1980.

[5] D. Sarason, Products of Toeplitz operators, in: Linear and Complex Analysis Problem Book 3, Part I, Lecture Notes in Math. 1573, Springer, Berlin, 1994, 318-319. 
[6] K. Stroethoff and D. C. Zheng, Products of Hankel and Toeplitz operators on the Bergman space, J. Funct. Anal. 169 (1999), 289-313.

[7] K. Stroethoff and D. C. Zheng, Bounded Toeplitz products on weighted Bergman spaces, J. Operator Theory 59 (2008), 277-308.

[8] - - - Bounded Toeplitz products on Bergman spaces of the unit ball, J. Math. Anal. Appl. 325 (2007), 114-129.

[9] D. C. Zheng, The distribution function inequality and products of Toeplitz and Hankel operators, J. Funct. Anal. 138 (1996), 477-501.

[10] K. H. Zhu, Spaces of Holomorphic Functions in the Unit Ball, Grad. Texts in Math. 226, Springer, New York, 2005.

Małgorzata Michalska, Maria Nowak, Paweł Sobolewski

Institute of Mathematics

Maria Curie-Skłodowska University

20-031 Lublin, Poland

E-mail: malgorzata.michalska@poczta.umcs.lublin.pl

mt.nowak@poczta.umcs.lublin.pl

pawel.sobolewski@umcs.eu

Received 8.9.2009

and in final form 6.2.2010 
